\title{
Anomalously bright UV lines of Fe II as a probe of gas condensations in the vicinity of hot stars
}

\author{
S. Johansson ${ }^{1}$ and V. S. Letokhov ${ }^{2,1}$ \\ ${ }^{1}$ Lund Observatory, Lund University, PO Box 43, 22100 Lund, Sweden \\ e-mail: Vladilen.Letokhov@astro.lu.se \\ 2 Institute of Spectroscopy, Russian Academy of Sciences, Troitsk, Moscow region 142190, Russia
}

Received 26 May 2003 / Accepted 13 October 2003

\begin{abstract}
Two UV lines of Fe II at 2507/2509 $\AA$ are extremely bright in spatially resolved HST/STIS spectra of dense gas condensations (the so called Weigelt blobs) close to the central star of $\eta$ Carinae. The lines also appear unresolved in HST/FOS spectra of the star AE And. Under normal conditions the lines should hardly be observable. Based on recent studies of the formation of the $\lambda \lambda 2507 / 2509$ lines as part of a radiative cycle driven by HLy $\alpha$ pumping and including stimulated emission we propose the possibility of using them as a probe of non-homogeneities in gas condensations located outside hot, massive stars.
\end{abstract}

Key words. atomic processes - line: formation - radiation mechanisms: non-thermal - stars: individual: $\eta$ Car, AE And

\section{Introduction}

We propose the possibility of using two ultraviolet Fe II lines at $2507 / 2509 \AA$ as a probe of spatial non-homogeneities in gas condensations (GC) outside hot, massive stars. Due to the anomalously high intensity of these particular Fe II lines in such GC's they will appear in the integrated spectrum of stars impossible to resolve spatially. The lines were first reported and identified in $I U E$ spectra of the symbiotic star V1016 Cyg (Nussbaumer \& Schild 1981) based on the extended laboratory analysis of Fe II (Johansson 1978). The excitation mechanism behind the lines in various objects was thoroughly discussed by Johansson \& Jordan (1984). The lines appeared as anomalously strong and narrow lines on top of a broad feature in IUE spectra of $\eta$ Car (Viotti et al. 1989).

The anomalous UV lines $\lambda \lambda 2507 / 2509$ occur as a result of selective photoexcitation of Fe II by $\mathrm{H}$ Ly $\alpha$ radiation due to an accidental wavelength coincidence (Johansson \& Jordan 1984; Johansson \& Hamann 1993). H Ly $\alpha$ coincides with a transition that combines a low-lying $(1 \mathrm{eV})$ excited state and two closely spaced, high lying states in Fe II, and the decay from these two levels produces the UV lines. The $\lambda \lambda 2507 / 2509$ lines have caused special attention lately due to their presence in spectra of $\eta$ Car, recorded at high spatial and spectral resolution (see review by Davidson \& Humphreys 1997) with the Hubble Space Telescope (HST).

The first direct observation of GC's in $\eta$ Car was made by speckle interferometry (Weigelt \& Ebersberger 1986), giving an image of the central star and three blobs. These blobs, later

Send offprint requests to: S. Johansson,

e-mail: sveneric.johansson@astro.lu.se called the Weigelt blobs, were shown to be located a few hundreds of $\mathrm{AU}$ from the central star. Drastically improved observational possibilities were offered by the high-resolution spectrometers (HRS and STIS) onboard the $H S T$, providing an angular resolution of $0.1^{\prime \prime}$ and a spectral resolution of $\lambda / \Delta \lambda \approx$ $10^{5}$ (Kimble et al. 1998). These instruments made it possible to observe the spectrum of the Weigelt blobs separately from the photospheric radiation of the central star itself. Analysis of the GHRS and STIS spectra have shown that the Weigelt blobs emit numerous narrow lines of many ionized elements (Zethson 2001). However, the most spectacular feature is the extraordinary bright fluorescence in the Fe II $\lambda \lambda 2507 / 2509$ lines (see Davidson et al. 1995, and later HST data by Gull et al. 2001).

Moreover, Davidson (2001) has pointed out the amazing similarity between the UV spectra of AE And in M 31 and the core of $\eta$ Car according to the data obtained by Szeifert et al. (1996). He emphasized that this intensity anomaly was at the time known only for these two stars and required special attention. The great attention to the mysterious Fe II lines, the mechanism behind their formation in $\eta$ Car, and their appearance in other objects have stimulated the present paper as a basis for future observations.

\section{Physical model of the formation of the Fe II $\lambda \lambda 2507 / 09$ lines in a blob}

An estimate (Hamann et al. 1999) of the column density of neutral hydrogen in a compact GC of $\eta$ Car, $n_{\mathrm{H}}=N_{\mathrm{H}} D$, yields a value in excess of $4 \times 10^{21} \mathrm{~cm}^{-2}$. It means that the GC is optically thick for the Lyman continuum $(\lambda<912 \AA)$, i.e.

$\tau_{\mathrm{c}}=\sigma_{\mathrm{ph}}\left(v_{\mathrm{c}}\right) N_{\mathrm{H}} D>>1$, 
where $\sigma_{\mathrm{ph}}\left(v_{\mathrm{c}}\right)$ is the photoionization absorption cross-section near the ionization limit, and $N_{\mathrm{H}}$ is the concentration of neutral hydrogen. Equation (1) can explain the formation of two separate regions in a blob: a hot $\mathrm{H}$ II region facing the star and a rear colder H I region. The two regions are separated by the Strömgren boundary located inside the blob. The H I region is responsible for a forest of narrow Fe II lines, which can mainly be grouped into two categories: fluorescence lines from high levels photoexcited by Ly $\alpha$ and forbidden lines from low, metastable levels (Zethson 2001).

As implied by Eq. (1), it is important that the blob hosts an $\mathrm{H} \mathrm{I}$ region, in which all $\mathrm{Fe}$ atoms, capable of being selectively photoexcited by $\operatorname{Ly} \alpha$, are ionized. This fact forms the basis for a physical model of the formation of the $\lambda \lambda 2507 / 09$ lines (Johansson \& Letokhov 2001b; Klimov et al. 2002) in the Weigelt blobs, located at a distance $R_{\mathrm{b}} \approx\left(10^{2}-10^{3}\right) r_{\mathrm{s}}$ from the central star in $\eta \mathrm{Car}$, where $r_{\mathrm{s}}$ is the stellar radius. Another important and critical requirement is that $R_{\mathrm{b}}$ is small enough to provide for a high spectral temperature of $\operatorname{Ly} \alpha, \mathrm{T}_{\alpha}$, at a frequency matching the Fe II pumping channel. $T_{\alpha}$ should be comparable with the effective temperature $T_{\mathrm{st}}$ of the star.

In principle, one can predict the value of $T_{\alpha}$ from estimates of the intensity of the Lyman continuum emitted by $\eta$ Car, the dilution factor, the influence of the stellar wind, etc. However, these estimates are quite uncertain, since the physical conditions in the immediate vicinity of the central star are not clearly understood. For this reason, it seems quite acceptable to estimate $T_{\alpha}$ from observational data, e.g. the anomalous brightness and behavior of the $\lambda \lambda 2507 / 09$ lines. With such an approach, the $\mathrm{Fe}^{+}$ions are utilized as a probe of the physical conditions in the neighborhood of $\eta$ Car, since the fluorescent lines of Fe II are very sensitive to the intensity of the $\operatorname{Ly} \alpha$ radiation.

In Fig. 1 we show a schematic diagram of the energy levels and transitions in Fe II involved in the formation of the $\lambda \lambda 2507 / 09$ lines. The selective photo excitation by Ly $\alpha$ transfers the ions from the metastable state 1 to the short-lived odd-parity states $4\left(5 \mathrm{p}{ }^{6} \mathrm{~F}_{9 / 2}^{0}, 4 \mathrm{p}^{4} \mathrm{G}_{9 / 2}^{0}\right)$. These levels decay to states 3 (preferentially to $\mathrm{c}^{4} \mathrm{~F}_{7 / 2}$, and to a lesser extent to $c^{4} \mathrm{~F}_{9 / 2}$ ), emitting the $\lambda \lambda 2507 / 09$ lines. At $T_{\alpha} \geq 12000 \mathrm{~K}$, the photoexcitation rate $W_{\text {exc }}(1 \rightarrow 4)$ exceeds the radiative decay rate of state 3 :

$W_{\mathrm{exc}}=A_{41}\left[\exp \left(\frac{h v_{14}}{k T_{\alpha}}\right)-1\right]^{-1} \geq \frac{1}{\tau_{3}} \approx 10^{3} \mathrm{~s}^{-1}$,

where $A_{41}=1.4 \times 10^{7} \mathrm{~s}^{-1}$ is the Einstein coefficient for the $4 \rightarrow 1$ transition. Thus, if Eq. (2) is satisfied, there will be an accumulation of $\mathrm{Fe}^{+}$ions in the "pseudo-metastable" levels $c^{4} \mathrm{~F}_{9 / 2,7 / 2}$. The lifetime of these levels is of the order of millisecond due to their relationship with the "pure" metastable levels of Fe II, but in the complex atomic structure they extend above the next higher configuration of opposite parity (state 2 in Fig. 1). Such an accumulation in "pseudometastable" states is thus unusual and it becomes an important ingredient in our model used to explain the origin of the anomalous $\lambda \lambda 2507 / 09$ lines. It should be emphasized that the hydrogen density $N_{\mathrm{H}}<<10^{13} \mathrm{~cm}^{-3}$, which means that collisions do not contribute to the depletion of the long-lived Fe II states.

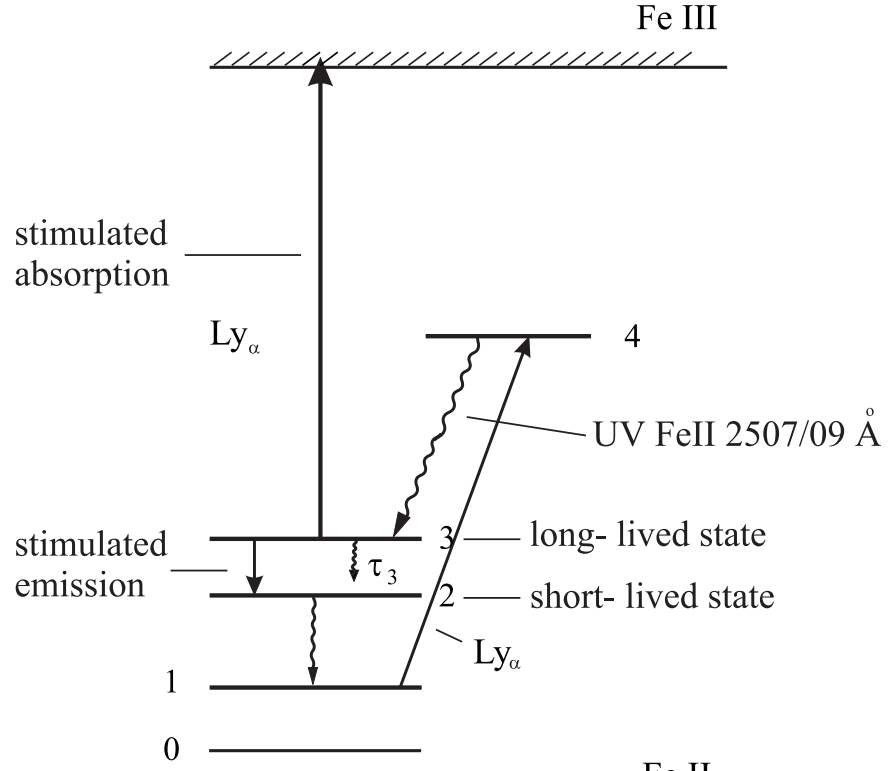

Fe II

Fig. 1. Schematic diagram of energy levels and transitions of Fe II, relevant to the radiative cycle $(1 \rightarrow 4 \rightarrow 3 \rightarrow 2$ ) proposed for the formation of the anomalously bright $\lambda \lambda 2507 / 09$ lines observed in UV spectra of $\eta$ Car and AE And. The lines form when the Ly $\alpha$ pumped state 4 decays to the long-lived state 3 . The intensity is built up due to collisionless depletion of the long-lived state in the form of both stimulated photoionization absorption and stimulated emission of radiation in the $3 \rightarrow 2$ transition.

In our initial model (Johansson \& Letokhov 2001a,b; Klimov et al. 2002), the accumulation of $\mathrm{Fe}^{+}$ions in the "pseudo-metastable" states leads to a noticeable optical thickness of the $\lambda \lambda 2507 / 09$ lines. The model is based on depletion of these levels by absorption, i.e. upward transitions stimulated by $\operatorname{Ly} \alpha$, as shown in Fig. 1. Such a depletion changes the optical density of the lines, assuming resonances in the photoionization cross section of Fe II from the "pseudo-metastable" states. However, in order to test this model one has to find these resonances experimentally, as the theoretical predictions of bound states of complex systems such as Fe II above the ionization limit are quite uncertain. We are therefore uncertain about the validity of these assumptions, and have suggested an additional and more plausible model.

In the advanced model (Johansson \& Letokhov 2003), we explore the fact that the accumulation of Fe II in the "pseudometastable" state 3 automatically leads to a population inversion, $\Delta N_{32}$, with respect to the short-lived states $2\left(\mathrm{z}^{4} \mathrm{D}_{7 / 2}^{0}\right.$ and $\mathrm{z}^{4} \mathrm{~F}_{9 / 2}^{0}$ ) in Fig. 1. With a sufficient size of the blob and density of Fe II, the resultant amplification increases the intensity of the spontaneous radiation due to stimulated downward transitions, occurring at a rate much in excess of their spontaneous decay rate. The analysis of the operation of such an astrophysical laser amplifier is beyond the scope of the present Letter and will be presented in another paper (Johansson \& Letokhov, in preparation). Let us stress that the processes suggested in the initial and advanced models as mechanisms for depletion 


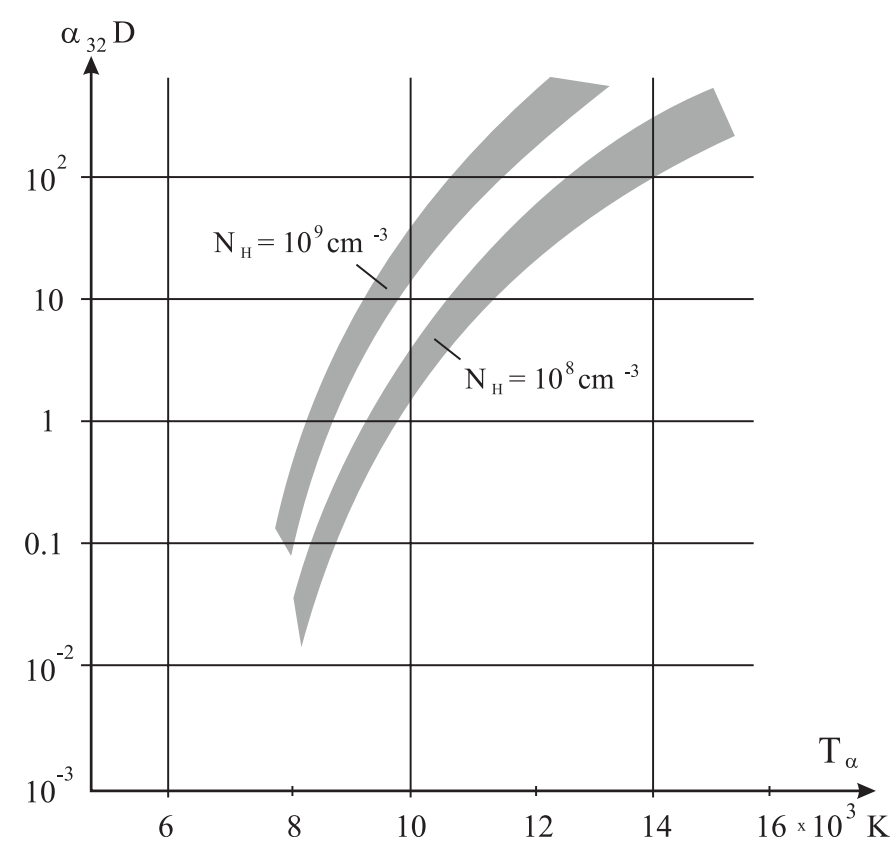

Fig. 2. Amplification $\left(\exp \left(\alpha_{32} D\right)\right)$ in the $3 \rightarrow 2$ transition in Fe II as a function of the effective spectral temperature $T_{\alpha}$ of the $\operatorname{Ly} \alpha$ radiation for two different values of the hydrogen concentration. Assumptions about the $\mathrm{Fe} / \mathrm{H}$ ratio are given in the text.

of the "pseudo-metastable" level 3 are complementary, i.e. they do not exclude each other.

We should emphasize that the population inversion $\Delta N_{32}=$ $N_{3}-N_{2}$ occurs always in a low-density plasma independent of the magnitude of $W_{\text {exc }}$, because of the short lifetime (3-4 ns) of the lower level 2 in the $3 \rightarrow 2$ transition. If Eq. (2) is satisfied, the population inversion is at maximum, but for $W_{\mathrm{exc}}<<1 / \tau_{3}$, the amplification coefficient $\alpha_{32}$ for the $3 \rightarrow 2$ transition is defined by

$\alpha_{32}=\sigma_{32} \Delta N_{32}, \quad$ where $\Delta N_{32}=W_{14}^{\mathrm{exc}} \tau_{3} N_{1}$.

The cross-section for the stimulated $3 \rightarrow 2$ transition is $\sigma_{32} \approx$ $(1.2-3.7) \times 10^{-16} \mathrm{~cm}^{2}$, and $N_{1}=f \cdot N_{\mathrm{Fe}}$ is the population in the initial state 1 , where $N_{\mathrm{Fe}} \approx 10^{-4} N_{\mathrm{H}}$ and $f$ is the fraction of $\mathrm{Fe}^{+}$ions in state 1. In Fig. 2 we show the value of the exponent $\left(\alpha_{32} D\right)$ in the amplification $K=\exp \left(\alpha_{32} D\right)$ of the $3 \rightarrow 2$ transition as a function of the spectral temperature of $\operatorname{Ly} \alpha, T_{\alpha}$, for two different values of $N_{0}$. The size of the blob is $D \approx 10^{15} \mathrm{~cm}$ and the population ratio is set to $f \approx 10^{-2}$. The uncertainty in the values of $\left(\alpha_{32} D\right)$ has its origin in the uncertainty of the temperature $T=100-1000 \mathrm{~K}$ in the $\mathrm{HI}$ region and the corresponding uncertainty in the Doppler width $\Delta v_{\mathrm{D}} \approx(200-600) \mathrm{MHz}$ of the amplified $3 \rightarrow 2$ transition. As can be seen from Eq. (3), a reduction of $N_{3}$ (the relative population of level 3) when $W_{\text {exc }}<<10^{3} \mathrm{~s}^{-1}$ at $T \leq$ $12000 \mathrm{~K}$, say, can be compensated for by increasing the concentration of Fe II to attain the necessary amplification coefficient (Johansson \& Letokhov 2003). For example, at $T_{\alpha}=$ $10000 \mathrm{~K}$ the excitation rate $W_{\text {exc }}=10^{2} \mathrm{~s}^{-1}$ and to attain a high amplification coefficient and the corresponding stimulated radiation intensity, the population $N_{1}$ in the initial state has to be increased ten times. This requirement is more soft than the one used in our previous work (Johansson \& Letokhov 2003). However, if $T_{\alpha}$ is below $8000-9000 \mathrm{~K}$ the reduced $W_{\text {exc }}$ can hardly be compensated for by a matching increase in $N_{1}$. Thus, within the framework of the alternative model of depletion of the "pseudo-metastable" states 3, the anomalous intensity of the $\lambda \lambda 2507 / 09$ Fe II lines can be explained by a radiative cycle in Fe II driven by Ly $\alpha$ and involving both spontaneous and stimulated radiative transitions.

\section{Bright UV Fe II lines from gas condensations}

The Ly $\alpha$ radiation responsible for the selective photoexcitation of Fe II can have its source in two regions: (1) the front, compact part of the GC that generates intense Ly $\alpha$ radiation as a result of recombination and (2) the illuminated part of the stellar wind that is moving away from the rear side of the GC. For this reason, we discuss both these possibilities.

The first source is the $\mathrm{H}$ II region produced by absorption of the Lyman continuum from the star, which is transferred into intense radiation in the immediate vicinity of the $\mathrm{H}$ I region containing Fe II. This source has been discussed earlier (Klimov et al. 2002). To compensate for the wavelength detuning of $2.4 \AA$ between Ly $\alpha$ and the absorbing Fe II channel by diffusion Doppler broadening, it is necessary that the optical density $\tau_{0}(\operatorname{Ly} \alpha)$ of the $\mathrm{H}$ I region is about $2 \times 10^{8}$ as estimated by Hamann et al. (1999). This value agrees with earlier estimates by Davidson (1997) based on the column density of H I and the size of the GC. At first glance the $\mathrm{H}$ I region acts like a blanket, reflecting the emerging Ly $\alpha$ photons back into the $\mathrm{H}$ II region, which has an open boundary towards the star. This question was briefly discussed in a work by Auer (1968), but we know nothing about any quantitative calculations concerning this effect.

The second source, which is toroidal-shaped due to the blocking by the GC itself, is appealing. Seen from the rear side of the blob it gives a redshift of $\operatorname{Ly} \alpha$ of the same size as the wavelength detuning between Ly $\alpha$ and the pumped Fe II transition. However, this occurs at the cost of a reduced amount of absorbed energy from the Lyman continuum and, hence, intensity of Ly $\alpha$ radiation. The optimal case is the one where the $\mathrm{H} \mathrm{II/H} \mathrm{I} \mathrm{boundary} \mathrm{for} \mathrm{the} \mathrm{stellar} \mathrm{wind} \mathrm{is} \mathrm{located} \mathrm{on} \mathrm{the} \mathrm{imme-}$ diate rear side of the GC, i.e. the Strömgren radius is somewhat longer than $R_{\mathrm{b}}$. In that case, the intensity of the Ly $\alpha$ radiation incident upon the GC would be at maximum. Any attempts to make quantitative estimates are very unreliable because the hydrogen density in the stellar wind in the vicinity of the GC is unknown. Even though the mass loss rate of $\eta$ Car is known (Davidson \& Humphreys 1997), the geometry of the stellar wind is aspherical and, probably, too complex in the immediate vicinity of $\eta$ Car.

Thus, the two potential Ly $\alpha$ sources have their pros and cons and require further investigation. Nevertheless, one can state that the anomalously bright $\lambda \lambda 2507 / 09$ Fe II lines are intrinsic characteristics of gas condensations, provided that the following two conditions are satisfied: (1) the hydrogen concentration in the GC is high enough for the Lyman continuum radiation to be blocked and a quasineutral $\mathrm{H}$ I region to 


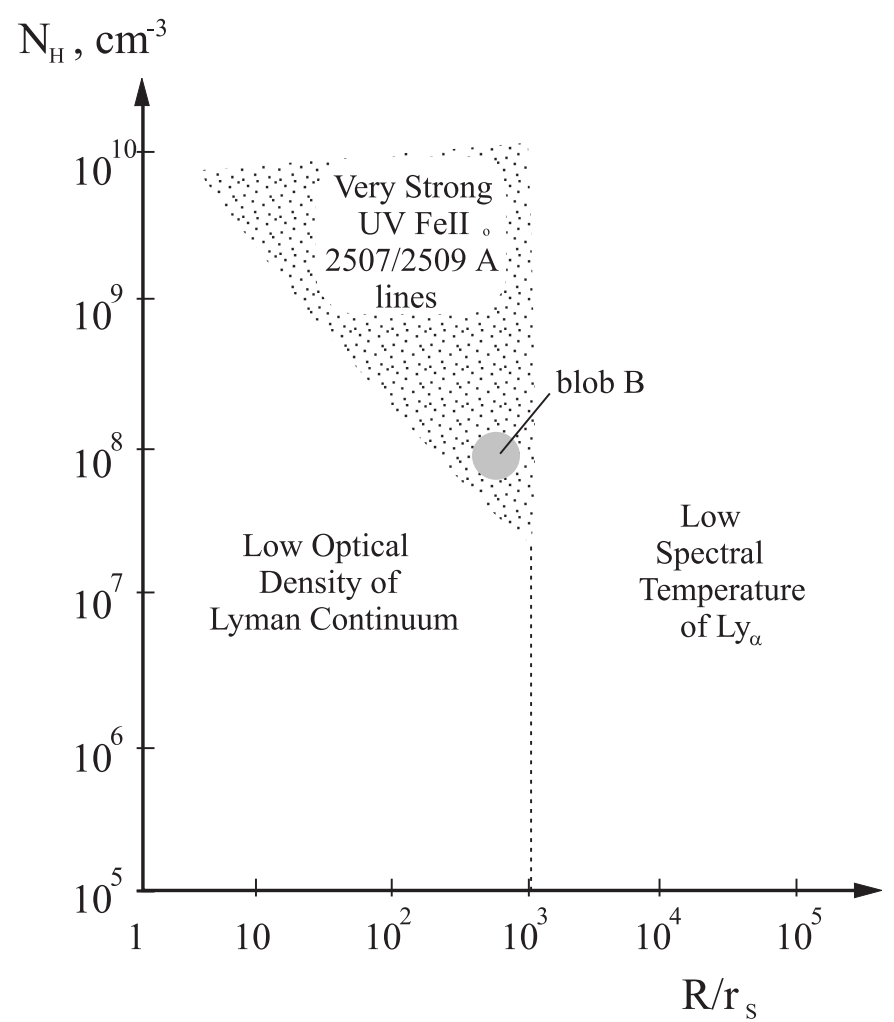

Fig. 3. A diagram of the hydrogen density versus the distance from the central star showing the parameter space in which the anomalously bright $\lambda \lambda 2507 / 09$ lines appear. Adequate parameter values for $\eta$ Car are given, and the parameter range for the gas condensations (blobs) close to are indicated.

be formed therein and (2) the GC is located close enough to the central star for an H I region to form near the GC (either within its front part or in the stellar wind region near its rear part) and emit intense $\operatorname{Ly} \alpha$ radiation (in the red wing of $\operatorname{Ly} \alpha$ ) capable of photoexciting Fe II in a selective way. These conditions are qualitatively illustrated in Fig. 3 by means of two main parameters - the distance $R_{\mathrm{b}}$ from the central star in terms of its photosphere radius $r_{\mathrm{s}}$ and the critical (minimal) hydrogen density $N_{\mathrm{H}}$ for the given distance $R$. The hydrogen density $N_{\mathrm{H}}$ should exceed some critical value $N_{0}^{\text {cr }}$ (Klimov et al. 2002):

$N_{0}^{\mathrm{cr}}=\left(\frac{I_{\mathrm{c}}}{\alpha D}\right)^{1 / 2}=\frac{r_{\mathrm{s}}}{L}\left(\frac{P\left(v_{\mathrm{c}}, T_{\mathrm{s}} \Delta v_{\mathrm{ph}}\right.}{\alpha D}\right)^{1 / 2}$,

where $\alpha$ is the rate constant coefficient of the $\mathrm{H} \mathrm{II} \rightarrow \mathrm{H}$ I recombination process. As in our previous paper (Johansson \& Letokhov 2001b), we make estimates by using the average black-body radiation intensity $P\left(v_{\mathrm{c}}\right)$ of $\eta$ Car with the effective temperature $T_{\mathrm{s}}$ and photoionization cross section $\sigma_{\mathrm{ph}}(v)$ values in the effective frequency interval $\Delta v_{\mathrm{ph}} \approx 3 \mathrm{eV}$ above $v_{\mathrm{c}}$, where $P(v)$ and $\sigma_{\mathrm{ph}}(v)$ are sufficiently large. Condition (4) means that the local Strömgren boundary separating the H II and $\mathrm{H} \mathrm{I}$ regions is inside the gas condensation. For blob $\mathrm{B}$ in $\eta$ Car, with $D \leq 10^{15} \mathrm{~cm}$ and $T_{\mathrm{s}}=30000 \mathrm{~K}$, the critical density $N_{0}^{\mathrm{cr}} \geq 10^{8} \mathrm{~cm}^{-3}$. The maximal distance $R$ is determined by the requirement of a minimum effective temperature of Ly $\alpha$, $T_{\alpha} \geq 10000 \mathrm{~K}$. This approximate value in Fig. 3 is based on observational data for the distance of blob B from $\eta$ Car (Davidson et al. 1997).

Davidson (2001) has pointed out the amazing similarity in the UV spectra around $2500 \AA$ between AE And in M 31 and $\eta$ Car and its surroundings. However, the spectrum of AE And represents integrated light of the whole object in contrast to the spatially resolved $H S T$ spectra of $\eta$ Car. To the left in Fig. 4 we show fragments of these spectra according to data from Davidson et al. (1995) and Szeifert et al. (1996). In both cases, the $\lambda \lambda 2507 / 09$ Fe II feature is very bright. Davidson (2001) emphasized that this intensity anomaly was at the time known only for these two stars and required special attention. In the light of our model developed for the appearance of these anomalous lines in the blobs near $\eta$ Car, whose main features are formulated above, it seems expedient to consider the case of AE And in M 31.

$\mathrm{AE}$ And is an extremely luminous blue star with a temperature probably as high as that of $\eta \mathrm{Car}\left(T_{\mathrm{s}} \approx 30000 \mathrm{~K}\right)$. The strength of the $\lambda \lambda 2507 / 09$ feature suggests that the immediate vicinity of $\mathrm{AE}$ And hosts gas condensations with a hydrogen column density $n_{\mathrm{H}}$ greater than the critical density, defined by condition (4).

Due to the huge distance AE And and its surroundings are observed in integrated light, and it is not possible to associate the radiation observed with any specific region of the object. This means that the intensity of the $\lambda \lambda 2507 / 09$ lines from possible gas condensations should exceed that from AE And itself. The lines from the gas condensations would otherwise be indiscernible from the stellar blackbody radiation in the same spectral interval.

In the HST/STIS spectra of $\eta$ Car, the GC's (the Weigelt blobs) are spatially resolved from the central star itself, which allows us to directly study the conditions where the $\lambda \lambda 2507 / 09$ lines are formed. In Fig. 4, however, we show for comparison a $0.5^{\prime \prime}$ spatial extract of he HST/FOS spectra of $\eta$ Car recorded with the $0.3^{\prime \prime}$ circular aperture (Davidson et al. 1995) and the FOS spectrum of AE And (see Fig. 3 in Szeifert et al. 1996). The similarity of these spectra around $2500 \AA$ may infer similarities in the plasmas where the $\lambda \lambda 2507 / 09$ lines are formed. For example, blob B of $\eta$ Car intercepts the radiation of the star within a significant solid angle of $\left(D / R_{\mathrm{b}}\right)^{2} \approx 0.07$, where the distance to the star is $R_{\mathrm{b}} \approx$ $4 \times 10^{15} \mathrm{~cm}$. Under these conditions intense $\lambda \lambda 2507 / 09$ lines can appear even against the stellar blackbody radiation. In the case of AE And, the angular size of a potential GC seen by the star the star should not be smaller than for the $\eta$ Car case.

Based on the model (Johansson \& Letokhov 2001a,b; 2003) developed for the formation of the $\lambda \lambda 2507 / 09$ lines in the GC's of $\eta$ Car, and the similarity between the spectra of $\eta$ Car and AE And in M 31 (Szeifert et al. 1996), one can consider the observation of very intense $\lambda \lambda 2507 / 09$ lines as a method for seeking such circumstellar gas condensations even without access to high spectral and spatial resolution. The anomalous brightness make the lines appear in spite of stellar background radiation. The spike seen in low resolution (Fig. 4) does not provide any information about the intensity ratio of the $\lambda \lambda 2507 / 09$ lines and their intensities relative to their satellite lines. These ratios will be different when the spectrum refers to a local region 


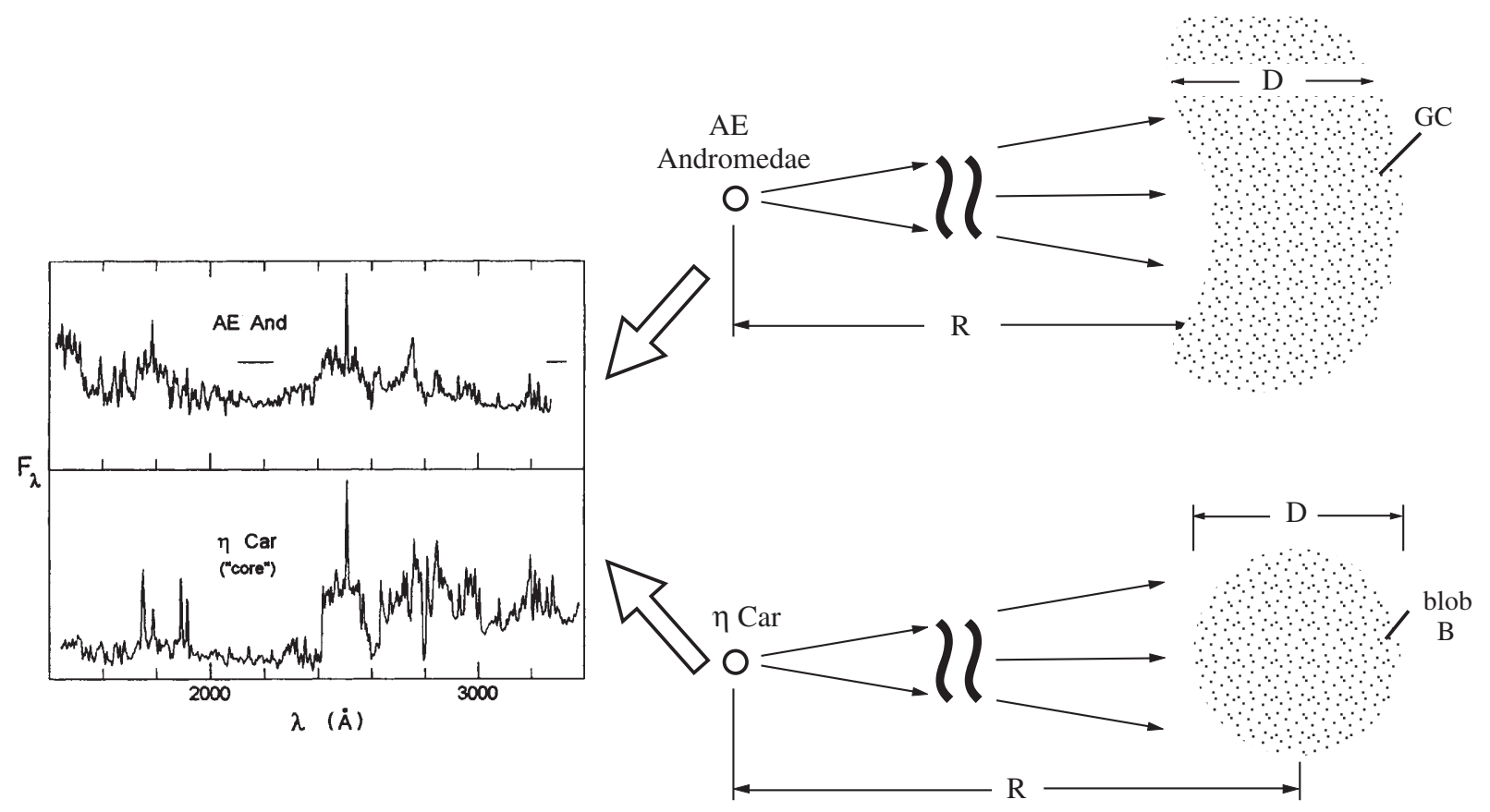

Fig. 4. Ultraviolet spectra of AE And in M 31 and a 0.5" spatial region of $\eta$ Car (adapted from Szeifert et al. 1996); right: geometry of the compact gas condensations illustrating the site of the anomalously bright $\lambda \lambda 2507 / 09$ lines.

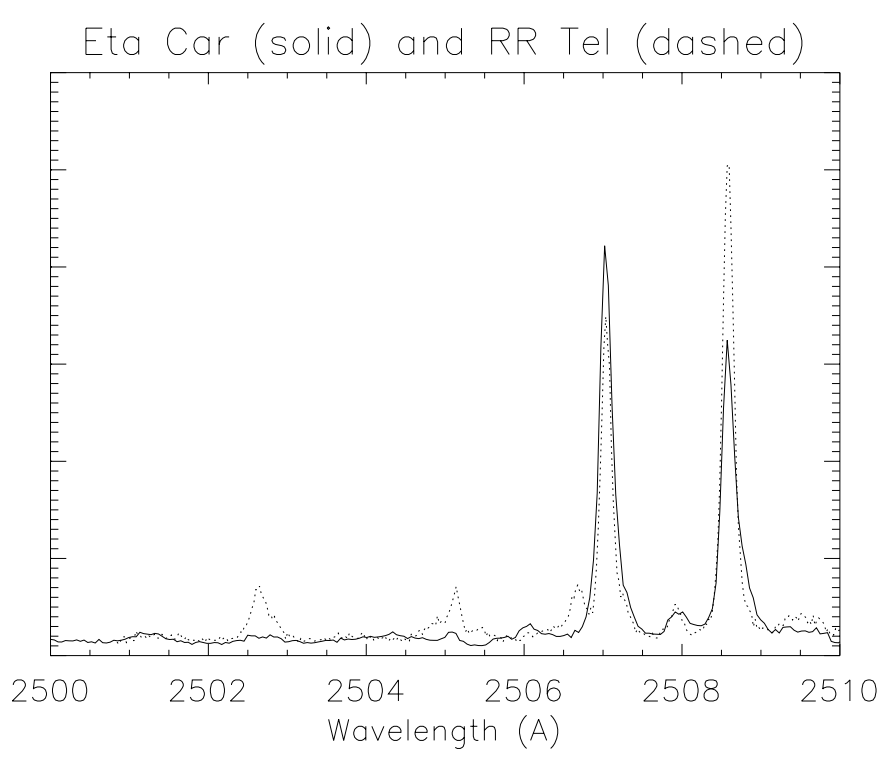

Fig. 5. High resolution HST/GHRS spectra around the $\lambda \lambda 2507 / 09$ lines of blob B in Eta Carinae (solid line) and of the symbiotic star RR Tel (dotted line).

spatially resolved from the central star (as is the case of the blobs in $\eta$ Car) and when the spectrum represents the whole extended object, i.e. radiation from the stellar photosphere and from the surrounding gas condensations. This is illustrated in Fig. 5 showing HST/GHRS spectra of two narrow spectral intervals (2500-2510 ̊) for $\eta$ Car and RR Tel. The difference is quite natural, because the cyclic interaction with stimulated emission most probably takes place in local zones, as in blob B of $\eta$ Car, and it will most probably be masked in integrated spectra of extended objects. However, a high spectral resolution would make it possible to observe not only these abnormally bright lines, but also the ratios of their intensities to those of their weak satellites, as was the case with $\eta$ Car (Hamann 1999). This would allow a better understanding of the similarity between the spectra of the $\lambda \lambda 2507 / 09$ lines in $\eta$ Car and AE And.

Acknowledgements. The authors thank H. Hartman for preparation of Fig. 5. The research project is supported by grants from the Swedish National Space Board (S.J.), The Royal Swedish Academy of Sciences and the Russian Foundation for Basic Research (\# 03-0216377) (V.L.).

\section{References}

Auer, L. H. 1968, ApJ, 153, 783

Davidson, K., Ebbets, D., Weigelt, et al. 1995, AJ, 109, 1784

Davidson, K., \& Humphreys, R. M. 1997, ARA\&A, 35, 1

Davidson, K. 2001, in Eta Carinae and Other Mysterious Stars: The Hidden Opportunities of Emission Line Spectroscopy, ed. T. R. Gull, S. Johansson, \& K. Davidson (San Fransisco: ASP), ASP Conf. Ser., 242, 3

Gull, T., Ishibashi, K., Davidson, K., \& Collins, N. 2001, in Eta Carinae and Other Mysterious Stars: The Hidden Opportunities of Emission Line Spectroscopy, ed. T. R. Gull, S. Johansson, \& K. Davidson (San Fransisco: ASP), ASP Conf. Ser., 242, 391

Hamann, F. W, Davidson, K., Ishibashi, H., \& Gull, T. 1999, in Eta Carinae at the Millenium, ed. J. A. Morse, R. M. Humphreys, \& A. Damineli (San Fransisco: ASP), ASP Conf. Ser., 179, 116 
Johansson, S. 1978, Phys. Scr., 18, 217

Johansson, S., \& Jordan, C. 1984, MNRAS, 210, 239

Johansson, S., \& Hamann, F. W. 1993, Phys. Scr., T47, 157

Johansson, S., \& Letokhov, V. S. 2001a, in Eta Carinae and Other

Mysterious Stars: The Hidden Opportunities of Emission Line Spectroscopy, ed. T. R. Gull, S. Johansson, \& K. Davidson (San Fransisco: ASP), ASP Conf. Ser., 242, 309

Johansson, S., \& Letokhov, V. S. 2001b, A\&A, 378, 266

Johansson, S., \& Letokhov, V. S. 2003, Phys. Rev. Lett., 90, 011101$1-4$
Kimble, R. I., Woodgate, B. E., Bowers, C. W., et al. 1998, ApJ, 492, L83

Klimov, V. V., Johansson, S., \& Letokhov, V. S. 2002, A\&A, 385, 313

Nussbaumer, H., \& Schild, H. 1981, A\&A, 101, 118

Szeifert, Th., Humphreys, R. M., Davidson, K., et al. 1996, A\&A, 314, 131

Viotti, R., Rossi, L., Cassatella, A., Altamore, A., \& Baratta, G. B. 1989, ApJS, 71, 983

Weigelt, G., \& Ebersberger, J. 1986, A\&A, 163, L5

Zethson, T. 2001, Ph.D. Thesis, Lund University, Lund, Sweden 